\title{
Similar transplant outcomes between haploidentical and unrelated donors after reduced-intensity conditioning with busulfan, fludarabine, and anti-thymocyte globulin in patients with acute leukemia or myelodysplastic syndrome
}

\author{
Mihong Choi ${ }^{1}$, Ja Yoon Heo ${ }^{1}$, Dong-Yeop Shin ${ }^{1,2,3}$, Ji Yun Lee ${ }^{4}$, Youngil Koh ${ }^{1,2,3}$, Junshik Hong ${ }^{1,2,3}$, \\ Inho Kim ${ }^{1,3}$, Sung-Soo Yoon ${ }^{1,2,3}$, Jeong-Ok Lee ${ }^{4}$, Soo-Mee Bang ${ }^{4}$ \\ ${ }^{1}$ Division of Hematology and Medical Oncology, Department of Internal Medicine, ${ }^{2}$ Center for Medical Innovation, Biomedical \\ Research Institute, Seoul National University Hospital, ${ }^{3}$ Cancer Research Institute, Seoul National University College of Medicine, \\ Seoul, ${ }^{4}$ Department of Internal Medicine, Seoul National University Bundang Hospital, Seongnam, Korea
}

p-ISSN 2287-979X / e-ISSN 2288-0011 https://doi.org/10.5045/br.2020.55.1.27 Blood Res 2020;55:27-34.

Received on July 27, 2019

Revised on December 12, 2019

Accepted on February 3, 2020
*This study was supported by the National Research Foundation of Korea (NRF) Grant funded by the Korean Government (MSIP) (No. NRF-2016R1A5A1011974).

\section{Correspondence to}

Dong-Yeop Shin, M.D., Ph.D. Division of Hematology and Medical Oncology, Department of Internal Medicine, Seoul National University College of Medicine, 101 Daehak-ro, Jongno-gu, Seoul 03080, Korea E-mail: stephano.dyshin@gmail.com (C) 2020 Korean Society of Hematology

\section{Background}

Although T-cell-replete hematopoietic cell transplantation (HCT) from haploidentical donors (HIDs) using anti-thymocyte globulin (ATG) has shown promising outcomes, previous studies often adopted heterogenous graft sources and conditioning.

\section{Methods}

We retrospectively compared HCT outcomes from 62 HIDs, 36 partially-matched unrelated donors (PUDs), and 55 matched unrelated donors (MUDs) in patients with acute leukemia or myelodysplastic syndrome using the same graft source of peripheral blood and a reduced intensity conditioning of busulfan, fludarabine, and ATG.

\section{Results}

The estimates of 3-yr disease-free survival (DFS) and overall survival (OS) rates were not significantly different among the MUD, HID, and PUD groups, at 46\%, "41\%, and 36\%" for the DFS rate $(P=0.844)$, and $55 \%, 45 \%$, and $45 \%$ for the OS rate $(P=0.802)$, respectively. Cumulative incidence of relapse and non-relapse mortality at $3 \mathrm{yr}$ was similar among different donor types. Subsequent multivariable analyses showed that the sex of the patient (male) and a high/very high disease risk index were independently associated with poorer DFS and OS, while the donor type was not.

\section{Conclusion}

T-cell replete HCT from HIDs using an ATG-containing reduced intensity conditioning regimen may be a reasonable option in the absence of matched related donors in patients with acute leukemia or myelodysplastic syndrome.

Key Words Haploidentical stem cell transplantation, HLA-matched unrelated donor, Reduced-intensity conditioning, Anti-thymocyte globulin

\section{INTRODUCTION}

For most high-risk hematologic malignancies, hematopoietic cell transplantation (HCT) has been the only curative method of treatment. The outcome of HCT is known to be best with matched related donors (MRDs), as the degree of human leukocyte antigen (HLA) match between the donor and the recipient correlates with the transplant outcome
$[1,2]$. However, MRDs are available only for a proportion of HCT candidates [1, 3]. For patients without a suitable $\mathrm{MRD}$, the next preferred option is to use matched unrelated donors (MUDs), followed by partially-matched unrelated donors (PUDs), haploidentical familial donors (HIDs), or cord blood units (CB). One may naturally wonder how the transplant outcomes from these alternative donors compare across the different donor types.

Although HIDs have the advantage of almost universal 
and immediate donor availability [4], the early experience of HCTs from HIDs was complicated by the manifestation of a strong bidirectional alloreactivity, e.g., graft failure and graft-vs.-host disease (GVHD) [5, 6]. These days, however, haploidentical HCTs have become increasingly popular with decades of efforts on better understanding of the immunologic mechanisms of HCT and developing measures to overcome the complications related to these [4, 7]. Indeed, T-cell-replete HCTs from HIDs using anti-thymocyte globulin (ATG) have demonstrated similar survival and acceptable risk of other morbidities compared to those of matched donors [8].

The overall picture is positive for HIDs, but most of the previous studies have compared different donor types adopting different graft sources, intensity of conditioning, and GVHD prophylaxis platforms. In other words, the influence of these factors on transplant outcomes cannot be excluded when interpreting their results.

Thus, the aim of this study was to compare T-cell-replete HCT outcomes from HIDs with those from PUDs and MUDs in a homogenous population with acute leukemia or myelodysplastic syndrome (MDS) who used the same graft source of peripheral blood and a reduced intensity conditioning (RIC) regimen of busulfan, fludarabine, and ATG.

\section{MATERIALS AND METHODS}

\section{Patient selection and treatment}

We retrospectively identified and collected clinical data from 153 adult patients with a diagnosis of acute leukemia or MDS who had undergone their first HCT from an unrelated or haploidentical donor with a specified ATG-containing RIC regimen between February 2008 and March 2017, at Seoul National University Hospital (SNUH) and Seoul National University Bundang Hospital (SNUBH). The donors included $10 / 10$ allele MUDs, $7 / 10$ or $8-9 / 10$ PUDs, or $3-4 / 6$ or $3-7 / 8$ or $6 / 10$ allele HIDs. Peripheral blood stem cells were used as the graft source for all cases. Patients who received HCT from CB or bone marrow as the stem cell source were excluded for their infrequency at these institutions during the study period. Conditioning consisted of intravenous busulfan at a dose of $3.2 \mathrm{mg} / \mathrm{kg}$ on D-7 and D-6, fludarabine at $30 \mathrm{mg} / \mathrm{m}^{2}$ from $\mathrm{D}-7$ to $\mathrm{D}-2$, and rabbit ATG (thymoglobulin) at $3.0 \mathrm{mg} / \mathrm{kg}$ from D-3 to D-1. Cyclosporin A or tacrolimus, with or without methotrexate, was used in all patients to prevent GVHD. The type and dose of immunosuppressant, dose of ATG, and the choice to switch to another calcineurin inhibitor were at the discretion of the treating physician. Methotrexate was used at a dose of $15 \mathrm{mg} / \mathrm{m}^{2}$ for $2 / 3 \mathrm{~d}$ or at $15 \mathrm{mg} / \mathrm{m}^{2} 2$ or 3 days the first day followed by $10 \mathrm{mg} / \mathrm{m}^{2}$ for the next $2 \mathrm{~d}$. The study protocol was reviewed and approved by the Institutional Review Board of SNUH and SNUBH, respectively.

\section{Definition}

Acute GVHD was diagnosed and graded clinically accord- ing to Glucksberg's criteria [9], and chronic GVHD according to the National Institutes of Health consensus criteria [10]. Relapse was defined by any evidence of the disease after $\mathrm{HCT}$, and non-relapse mortality (NRM) was defined as death from any cause except relapse. The HCT comorbidity index (HCT-CI) was applied according to the previous study by Sorror et al. [11]. The disease risk index (DRI) consisted of disease and stage risk, each of which was derived from a diagnosis with the cytogenetics data and remission status at the time of HCT, respectively, and has been shown to successfully risk stratify heterogenous allogeneic transplant recipients [12]. For the present study, the DRI was collapsed to a 2-group system of low/intermediate and high/very high risk, as proposed by the original study [12].

Statistical endpoints included GVHD-free, relapse-free survival (GRFS), disease-free survival (DFS), and overall survival (OS). GRFS is a composite endpoint encompassing ongoing morbidity from GVHD in addition to relapse and death $[13,14]$. GRFS events were defined grade III-IV acute GVHD chronic GVHD requiring systemic immunosuppressive treatment, relapse, or death. DFS was calculated as the time from HCT to relapse or death, and OS was defined as the time from HCT to death.

\section{Statistical methods}

Patient and treatment characteristics were compared with the chi-square test for categorical variables and one-way ANOVA for continuous variables. Survival curves were plotted using the Kaplan-Meier method and compared using the log-rank test. Cumulative incidence was estimated for individual failure events and compared using Gray's test, with death without GVHD being a competing risk for GVHD, relapse for NRM, and NRM for relapse. The Cox proportional hazards model was applied to identify risk factors significantly related to survival, in which variables with statistical significance from univariable analyses were subsequently used to construct multivariable analyses, along with the donor types. All $P$-values were two-sided and values of $<0.05$ were considered statistically significant. Statistical analyses were performed using two statistical software packages: SPSS version 23.0 (IBM Corp, Armonk, NY, USA) and R 3.5.1.

\section{RESULTS}

\section{Baseline characteristics}

The characteristics of the included 153 patients are shown in Table 1. Overall, the median age of the recipients was $52 \mathrm{yr}$ (range, 16-68) and 52.3\% of the patients were males. Median HCT-CI scores were 0 in both overall and each of the three donor groups. The most common diagnosis was acute myeloid leukemia (AML, 59.5\%), followed by acute lymphoblastic leukemia (ALL, 26.1\%), and MDS (14.4\%). DRI was low/intermediate in $75.2 \%$ and high/very high in $24.8 \%$ of the patients. Patients received HCT after a median of 5.80 months (range, 2.07-197.43 mo) from their diagnosis 
Table 1. Patients and treatment characteristics.

\begin{tabular}{|c|c|c|c|c|c|}
\hline \multirow{2}{*}{ Variables } & \multirow{2}{*}{$\begin{array}{c}\text { Overall } \\
(\mathrm{N}=153)\end{array}$} & \multicolumn{4}{|c|}{ By donor type } \\
\hline & & $\mathrm{HID}(\mathrm{N}=62)$ & PUD $(\mathrm{N}=36)$ & MUD $(\mathrm{N}=55)$ & $P^{\mathrm{a})}$ \\
\hline Age, median (range) & $52(16-68)$ & $55(16-66)$ & $53(25-68)$ & $48(18-67)$ & 0.970 \\
\hline Sex, N (\%) & & & & & 0.647 \\
\hline Male & $80(52.3 \%)$ & $34(54.8 \%)$ & $20(55.6 \%)$ & $26(47.3 \%)$ & \\
\hline Female & $73(47.7 \%)$ & $28(45.2 \%)$ & $16(44.4 \%)$ & $29(52.7 \%)$ & \\
\hline HCT-Cl total scores, median (range) & $0\left(0^{-5}\right)$ & $0\left(0^{-5}\right)$ & $0(0-3)$ & $0(0-4)$ & 0.134 \\
\hline Disease, $N(\%)$ & & & & & 0.443 \\
\hline $\mathrm{AML}$ & $91(59.5 \%)$ & $36(59.7 \%)$ & $26(72.2 \%)$ & $28(50.9 \%)$ & \\
\hline MDS & $22(14.4 \%)$ & $8(12.9 \%)$ & $4(11.1 \%)$ & $10(18.2 \%)$ & \\
\hline ALL & $40(26.1 \%)$ & $17(27.4 \%)$ & $6(16.7 \%)$ & $17(30.9 \%)$ & \\
\hline Disease risk index & & & & & 0.379 \\
\hline Low to intermediate & $115(75.2 \%)$ & $43(69.4 \%)$ & $28(77.8 \%)$ & $44(80.0 \%)$ & \\
\hline High to very high & $38(24.8 \%)$ & $19(30.6 \%)$ & $8(22.2 \%)$ & $11(20.0 \%)$ & \\
\hline Time from diagnosis to HCT in mo, median (range) & $5.80(2.07-197.43)$ & $5.08(2.07-50.70)$ & $6.82(2.73-20.40)$ & $5.20(2.53-197.43)$ & 0.877 \\
\hline Female donor-to-male patient, $\mathrm{N}(\%)$ & $24(15.7 \%)$ & $12(19.4 \%)$ & $6(16.7 \%)$ & $6(10.9 \%)$ & 0.448 \\
\hline ABO incompatibility & & & & & 0.242 \\
\hline Matched & $60(39.2 \%)$ & $29(46.8 \%)$ & $9(25.0 \%)$ & $22(40.0 \%)$ & \\
\hline Major mismatch & $38(24.8 \%)$ & $12(19.4 \%)$ & $12(33.3 \%)$ & $14(25.5 \%)$ & \\
\hline Minor mismatch & $32(20.9 \%)$ & $14(22.6 \%)$ & $6(16.7 \%)$ & $12(21.8 \%)$ & \\
\hline Bidirectional & $22(14.4 \%)$ & $6(9.7 \%)$ & $9(25.0 \%)$ & $7(12.7 \%)$ & \\
\hline Infused CD $34^{+}$cells in $\times 10^{6} / \mathrm{kg}$, median (range) & $5.57(0.97-12.68)$ & $5.70(1.80-11.40)$ & $4.45(1.21-10.69)$ & $5.83(0.97-12.68)$ & 0.055 \\
\hline ATG total dose in $\mathrm{mg} / \mathrm{kg}$, median (range) & $9.00\left(4.5^{-12.0}\right)$ & $9.00(4.50-12.00)$ & $9.00(4.50-12.00)$ & $9.00(4.50-12.00)$ & 0.423 \\
\hline $\mathrm{CNI}$ use & & & & & 0.034 \\
\hline Cyclosporin A & 79 (51.6\%) & 27 (43.5\%) & $25(69.4 \%)$ & 27 (49.1\%) & \\
\hline Tacrolimus & $64(41.8 \%)$ & $28(45.2 \%)$ & $9(25.0 \%)$ & 27 (49.1\%) & \\
\hline Switch & $10(6.5 \%)$ & 7 (11.3\%) & $2(5.6 \%)$ & $1(1.8 \%)$ & \\
\hline Methotrexate use & & & & & $<0.001$ \\
\hline Yes & $95(62.1 \%)$ & 56 (90.3\%) & 19 (52.8\%) & 20 (36.4\%) & \\
\hline No & $58(37.9 \%)$ & $6(9.7 \%)$ & $17(47.2 \%)$ & $35(63.6 \%)$ & \\
\hline
\end{tabular}

of hematologic malignancy, and approximately $15.7 \%$ of the cases consisted of a male recipient and a female donor. CD34 cells were infused at a median dose of $5.57 \times 10^{6} / \mathrm{kg}$ (range, $0.97-12.68 \times 10^{6} / \mathrm{kg}$ ), and the median total ATG dose was 9.00 $\mathrm{mg} / \mathrm{kg}$ (range, $4.5-12.0 \mathrm{mg} / \mathrm{kg}$ ). Although these characteristics were comparable among groups with different donor types, cyclosporin A was more frequently used, compared to tacrolimus, in the PUD group than in the HID and MUD groups, at $69.4 \%$ vs. $43.5 \%$ and $49.1 \%$, respectively ( $P=0.034$ ). Additionally, the proportion of patients who used methotrexate was higher in the HID group than in the PUD and MUD group, at $90.3 \%$ vs. $52.8 \%$ and $36.4 \%$, respectively $(P<0.001)$.

\section{Estimate of survival}

In the total population, the 3-yr GRFS, DFS, and OS rates were $24.3 \%$ [95\% confidence interval (CI), 20.7-27.9\%], $42.0 \%$ (95\% CI, 37.8-46.2\%), and 49.1\% (95\% CI, $44.7-$ $53.5 \%$ ), respectively (Fig $1 \mathrm{~A}-\mathrm{C}$ ).

When grouped by donor type, the 3-yr GRFS rates were estimated to be $28.6 \%$ (95\% CI, 22.8-34.4\%), $17.5 \%$ (95\% CI, $10.6-24.4 \%$ ), and $23.2 \%$ (95\% CI, $17.2-29.2 \%$ ) for the HID, PUD, and MUD groups, respectively (Fig. 1D). The estimates of the DFS and OS rates at $3 \mathrm{yr}$ were numerically higher in the MUD group than in the HID and PUD groups, with $45.5 \%$ (95\% CI, $38.5-52.5 \%$ ) vs. $41.2 \%$ (95\% CI, $34.5-$ $47.9 \%$ ) and $36.3 \%$ (95\% CI, 27.5-45.1\%) for the DFS rates, and $55.1 \%$ (95\% CI, $48.2-62.0 \%)$ vs. $44.9 \%$ (95\% CI, 37.6$52.2 \%$ ) and $44.9 \%$ (95\% CI, 35.9-53.9\%) for the OS rates, respectively (Fig. 1E, F). There was no significant difference in the curves for GRFS $(P=0.438)$, DFS $(P=0.844)$, and OS $(P=0.802)$ among the three different donor types, based on log-rank tests (Fig. 1D-F).

When compared between groups with a different DRI, the 3-yr GRFS rates were estimated at 25.2\% (95\% CI, 20.929.5\%) and $21.1 \%$ (95\% CI, 14.5-27.7\%) for the low/intermediate and high/very high groups, respectively. There was no significant difference between the curves for GRFS $(P=0.189$; Fig. $1 \mathrm{G})$. In contrast, the DFS and OS curves differed by DRI groups $(P=0.016$ for DFS and 0.007 for OS; 


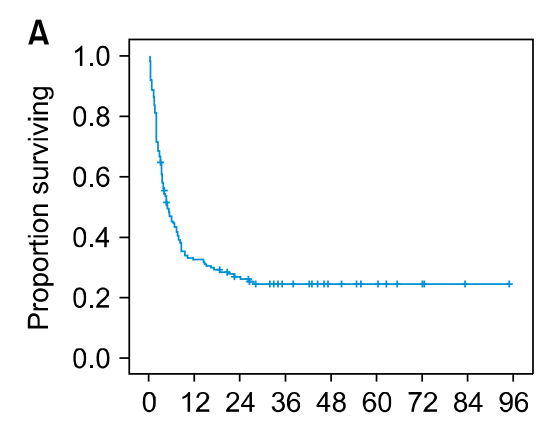

GVHD-free, relapse-free survival in months
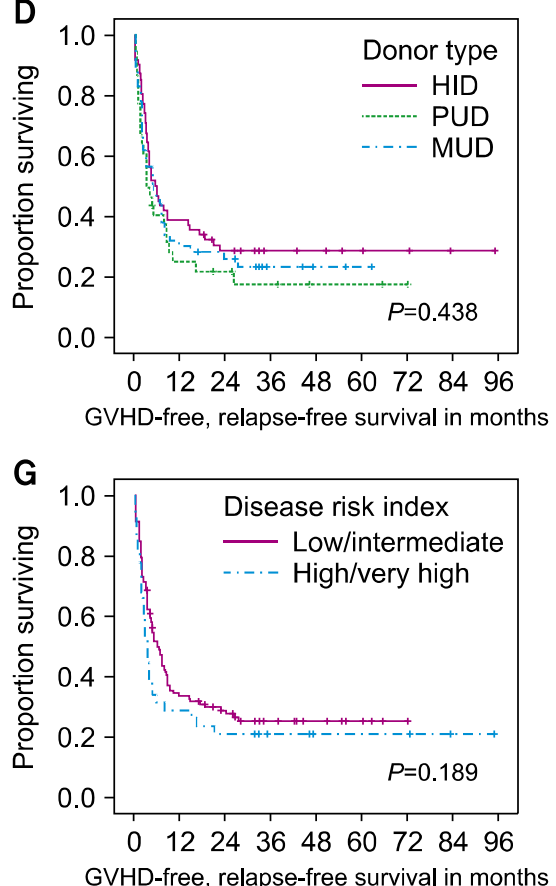
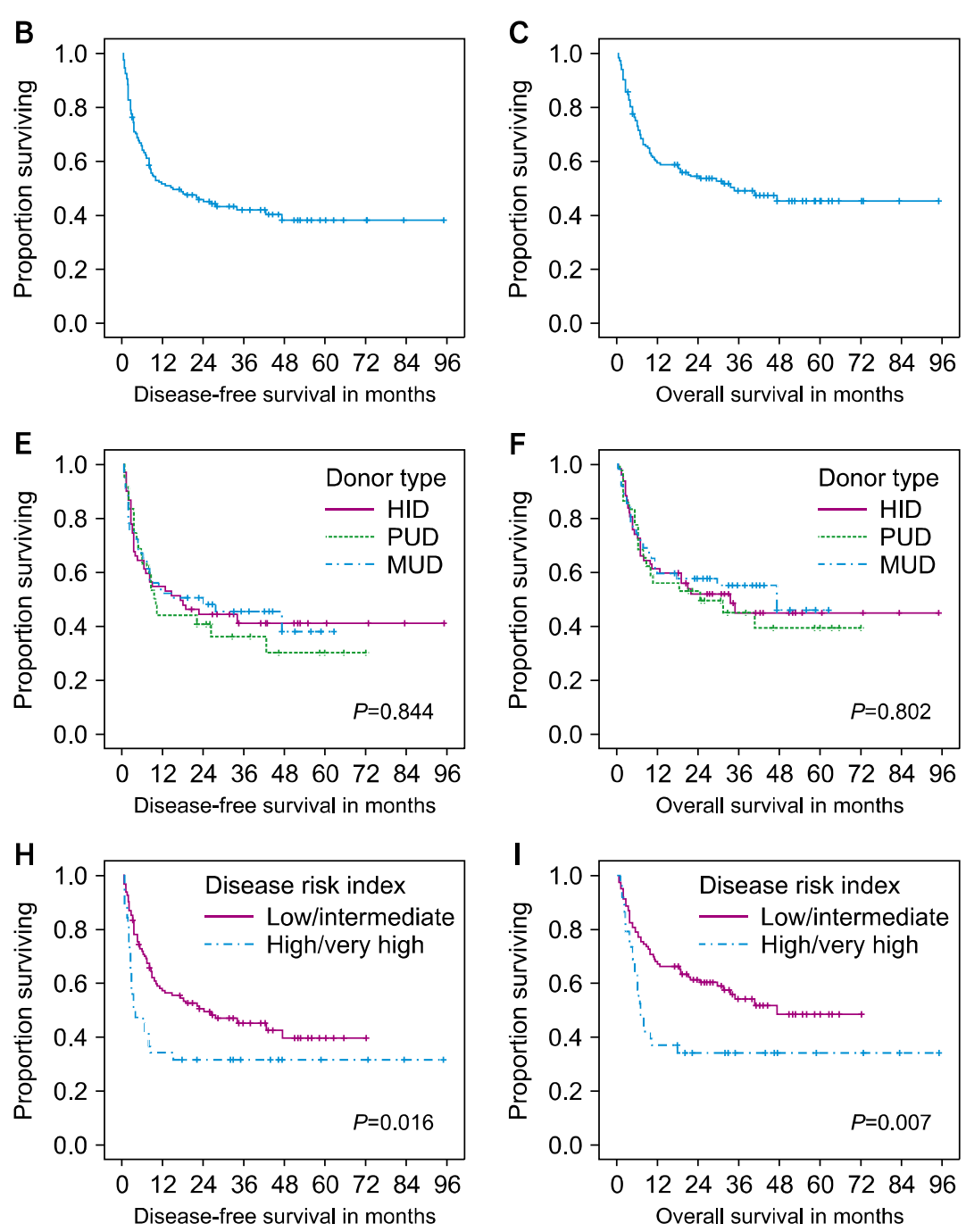

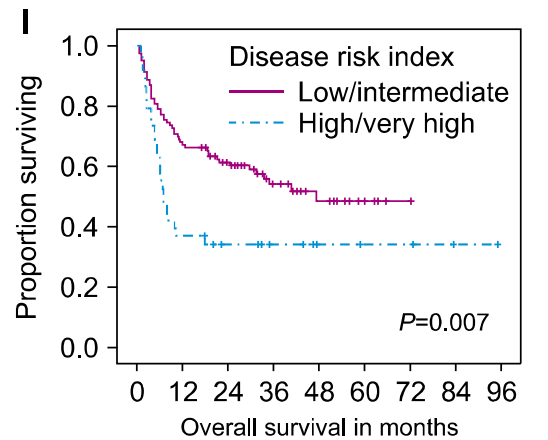

Fig. 1. Survival curves for the overall population. Graft-vs.-host disease (GVHD)-free, relapse-free survival (A). Disease-free survival (B). Overall survival (C). GVHD-free, relapse-free survival by donor types (D). Disease-free survival by donor types (E). Overall survival by donor types (F). GVHD-free, relapse-free survival by disease risk index $(\mathrm{G})$. Disease-free survival by disease risk index $(\mathrm{H})$. Overall survival by disease risk index $(\mathrm{I})$. Abbreviations: HID, haploidentical familial donors; MUD, matched unrelated donors; PUD, partially matched unrelated donors.

Fig. 1H, I). The estimates of the 3-yr DFS rates were $40.2 \%$ (95\% CI, 40.2-50.2\%) and 31.6\% (95\% CI, 24.1-39.1\%) for the low/intermediate and high/very high groups, respectively (Fig. 1H). The 3-yr OS rates were 54.0\% (95\% CI, 48.8-59.2\%) and $34.0 \%$ (95\% CI, 26.3-41.7\%) for the low/intermediate and high/very high groups, respectively (Fig. 1I).

\section{Cumulative incidence of individual failure events}

Grade III-IV acute GVHD was more frequently noted in the PUD group than in the other two groups, with a 100-d cumulative incidence of $19.4 \%$ (95\% CI, 12.7-26.2\%) vs. $8.1 \%$ (95\% CI, $4.6-11.6 \%$ ) and $9.1 \%$ (95\% CI, 5.2-13.0\%) for the PUD vs. HID and MUD groups, respectively (Fig. 2A). The incidence of chronic GVHD requiring systemic treatment was higher in the HID and MUD groups than in the PUD group, with a cumulative incidence of $34.3 \%$ (95\% CI, 28.1-40.4\%) and 25.9\% (95\% CI, 19.9-32.0\%) vs.
17.6\% (95\% CI, 10.9-24.3\%) at $3 \mathrm{yr}$, respectively (Fig. 2B). These differences in number, however, were not statistically significant ( $P=0.213$ for acute GVHD; $P=0.261$ for chronic GVHD).

The cumulative incidence of relapse was similar among different donor types, with 34.4\% (95\% CI, 28.3-40.6\%), $37.5 \%$ (95\% CI, 28.4-46.5\%), and 39.8\% (95\% CI, 32.8 $46.7 \%)$ at $3 \mathrm{yr}$ for HIDs, PUDs, and MUDs, respectively $(P=0.920$; Fig. 2C). NRM was not significantly different between the HID, PUD, and MUD groups, with 21.1\% (95\% CI, $15.9-26.4 \%$ ), 29.5\% (95\% CI, 18.6-34.0\%), and 14.7\% (95\% CI, 9.8-19.6) at 3 yr post-HCT, respectively $(P=0.594$; Fig. 2D). Relapse was observed later than 2 yr after HCT, whereas most NRM events in this study occurred within 1 yr after HCT. 

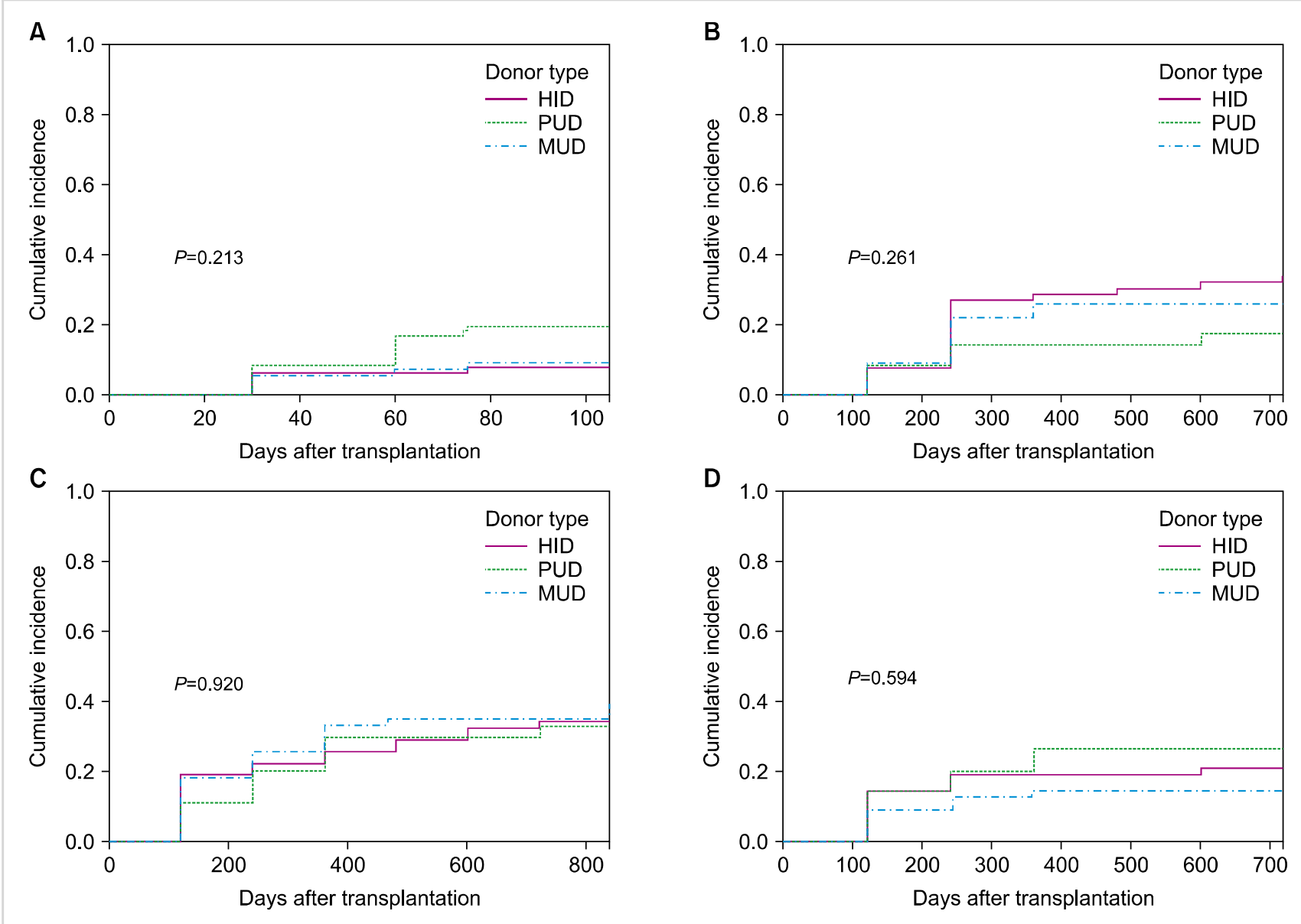

Fig. 2. Cumulative incidence curves by donor type. Acute graft-vs.-host disease of grade III-IV (A). Chronic graft-vs.-host disease requiring systemic treatment (B). Relapse (C). Non-relapse mortality (D).

Abbreviations: HID, haploidentical familial donors; MUD, matched unrelated donors; PUD, partially matched unrelated donors.

\section{Prognostic factor analysis for survival}

Of the clinical and treatment variables analyzed in univariable analyses, female donor-to-male recipient and no methotrexate use (vs. use) were associated with a significantly worse GRFS. Patient sex (female vs. male) and a higher dose of infused $\mathrm{CD} 34^{+}$cells were associated with a better DFS and OS, while higher HCT-CI scores and high/very high DRI (vs. low/intermediate) were associated with a worse DFS and OS (Table 2).

Subsequent multivariable analyses showed that female donor-to-male recipient and no methotrexate use (vs. use) were independently associated with a significantly worse GRFS, with a hazard ratio (HR) of 1.82 (95\% CI, 1.12-2.97; $P=0.015)$ and 1.58 (95\% CI, 1.02-1.58; $P=0.040)$, respectively. Patient sex (female vs. male) was associated with a better DFS and OS with an HR of 0.60 (95\% CI, 0.39-0.93; $P=0.024)$ for DFS and 0.56 (95\% CI, 0.35-0.90; $P=0.017)$ for OS. A high/very high (vs. low/intermediate) DRI was associated with a worse DFS and OS, with an HR of 1.77 (95\% CI, 1.11-2.83; $P=0.017$ ) for DFS and 1.94 (95\% CI, 1.19-3.18; $P=0.008$ ) for OS (Table 3). Donor type was not associated with survival in both univariable and multivariable analyses.

\section{DISCUSSION}

In this study, we found that GRFS, DFS, and OS were similar between HIDs, PUDs, and MUDs, but DFS and OS differed by patient sex and DRI in our cohort of HCT recipients with acute leukemia or MDS who used the same graft source of peripheral blood and the same RIC regimen of busulfan, fludarabine, and ATG. NRM tended to be higher with a mismatched donor than with a matched donor in this population, although without statistical significance.

Our estimates for alternative donor HCT for adult patients with acute leukemia closely followed those recently reported, based on a large registry of the European Society for Blood and Marrow Transplantation. Piemontese et al. [15] analyzed 3,568 recipients of alternative donor HCT from 2007 to 2013 for acute leukemia in their first or second remission. DFS and OS were longer with MUD than with HID and PUD, at $50 \%$ vs. $41 \%$ and $46 \%$ for $3-y$ DFS rates and $56 \%$ vs. $46 \%$ and $48 \%$ for 3 -yr OS rates. NRM at 3 yr was higher with HID and PUD than with MUD, at $29 \%$ and $29 \%$ vs. $21 \%$, respectively. GRFS rates and relapse rates at $3 \mathrm{yr}$ were similar among these donor types, at $33-36 \%$ and $25-30 \%$, 
Table 2. Univariable analyses for survival.

\begin{tabular}{|c|c|c|c|c|c|c|c|}
\hline Parameters & N & $\begin{array}{c}\text { Hazard ratio for GRFS } \\
(95 \% \mathrm{Cl})\end{array}$ & $P$ & $\begin{array}{l}\text { Hazard ratio for DFS } \\
\qquad(95 \% \mathrm{Cl})\end{array}$ & $P$ & $\begin{array}{l}\text { Hazard ratio for OS } \\
\qquad(95 \% \mathrm{Cl})\end{array}$ & $P$ \\
\hline Age & 153 & $0.99(0.98-1.01)$ & 0.421 & $0.99(0.98-1.01)$ & 0.345 & $1.00(0.98-1.02)$ & 0.983 \\
\hline \multicolumn{8}{|l|}{ Sex } \\
\hline Male & 80 & 1 & & 1 & & 1 & \\
\hline Female & 73 & $0.74(0.51-1.07)$ & 0.106 & $0.57(0.37-0.87)$ & 0.009 & $0.54(0.34-0.86)$ & 0.010 \\
\hline \multicolumn{8}{|c|}{ Female-to-male combination } \\
\hline No & 129 & 1 & & 1 & & 1 & \\
\hline Yes & 24 & $1.69(1.05-2.72)$ & 0.031 & $1.54(0.89-2.65)$ & 0.120 & $1.55(0.86-2.77)$ & 0.143 \\
\hline \multicolumn{8}{|l|}{ Diagnosis } \\
\hline AML & 91 & 1 & & 1 & & 1 & \\
\hline MDS & 40 & $0.72(0.46-1.12)$ & 0.145 & $1.04(0.63-1.70)$ & 0.889 & $0.93(0.54-1.58)$ & 0.784 \\
\hline ALL & 22 & $0.71(0.40-1.26)$ & 0.243 & $1.11(0.601-2.03)$ & 0.747 & $0.88(0.44-1.73)$ & 0.702 \\
\hline HCT-Cl scores & 153 & $1.09(0.89-1.33)$ & 0.406 & $1.28(1.04-1.58)$ & 0.021 & $1.32(1.06-1.65)$ & 0.014 \\
\hline \multicolumn{8}{|l|}{ Disease risk index } \\
\hline Low/intermediate & 115 & 1 & & 1 & & 1 & \\
\hline High/very high & 38 & $1.32(0.870-2.01)$ & 0.191 & $1.75(1.11-2.78)$ & 0.017 & $1.93(1.19-3.12)$ & 0.008 \\
\hline \multicolumn{8}{|l|}{ Timing of transplant } \\
\hline 2013-2018 & 140 & 1 & & 1 & & 1 & \\
\hline $2008-2012$ & 13 & $1.00(0.51-1.98)$ & 0.998 & $1.06(0.51-2.21)$ & 0.868 & $1.35(0.648-2.82)$ & 0.421 \\
\hline \multicolumn{8}{|l|}{ Donor type } \\
\hline MUD & 55 & 1 & & 1 & & 1 & \\
\hline HID & 62 & $0.86(0.56-1.31)$ & 0.481 & $1.03(0.63-1.68)$ & 0.911 & $1.12(0.66-1.89)$ & 0.681 \\
\hline PUD & 36 & $1.17(0.72-1.89)$ & 0.452 & $1.17(0.67-2.03)$ & 0.579 & $1.22(0.67-2.22)$ & 0.512 \\
\hline $\mathrm{CD} 4^{+}$cells, $\times 10^{6} / \mathrm{kg}$ & 153 & $0.98(0.91-1.05)$ & 0.505 & $0.91(0.83-0.99)$ & 0.032 & $0.90(0.82-0.99)$ & 0.030 \\
\hline ATG total dose & 153 & $1.02(0.93-1.13)$ & 0.622 & $1.05(0.94-1.17)$ & 0.429 & $1.09(0.97-1.23)$ & 0.166 \\
\hline \multicolumn{8}{|l|}{ Calcineurin inhibitor use } \\
\hline Cyclosporin A & 79 & 1 & & 1 & & 1 & \\
\hline Tacrolimus & 64 & $0.80(0.54-1.18)$ & 0.254 & $1.10(0.71-1.72)$ & 0.667 & $0.86(0.53-1.40)$ & 0.547 \\
\hline \multicolumn{8}{|l|}{ Methotrexate use } \\
\hline Yes & 95 & 1 & & 1 & & 1 & \\
\hline No & 58 & $1.47(1.01-2.14)$ & 0.042 & $1.21(0.79-1.85)$ & 0.389 & $1.04(0.67-1.65)$ & 0.865 \\
\hline
\end{tabular}

Abbreviations: ALL, acute lymphoblastic leukemia; AML, acute myeloid leukemia; ATG, antithymocyte globulin; DFS, disease-free survival; GRFS, graft-vs.-host disease-free, relapse-free survival; HCT-Cl, hematopoietic cell transplantation comorbidity index; HID, haploidentical familial donors; MDS, myelodysplastic syndrome; MUD, matched unrelated donors; OS, overall survival; PUD, partially-matched unrelated donors.

respectively [15].

Although our study was comparable to that of Piemontese et al. [15] to some extent, several differences make a direct comparison between these two studies difficult. Patients with advanced disease were not included, and myeloablative conditioning was given in more than half of the cases in the above-mentioned study. In addition, different graft sources and GVHD prophylaxis platforms were used according to different donor types, with HCT from HIDs adopting a higher proportion of bone marrow as the graft source and PTCy for GVHD prophylaxis.

With such diverse factors involved, heterogeneity has been inherent between studies on the outcome of allogeneic HCT. In this context, DRI was devised to risk-stratify recipients of allogeneic HCT by their disease type and status, the strongest determinants of survival in this setting, which was shown to be stable across different age groups, conditioning intensity, graft source, and donor type [11, 12]. McCurdy et al. [16] reported a risk-stratified outcome with HCT from
HIDs using non-myeloablative conditioning and PTCy, as most cases used for the refinement of DRI were recipients of matched donor HCT. Low, intermediate, and high/very high groups demonstrated $65 \%, 37 \%$, and $22 \%$ for the DFS rate at $3 \mathrm{yr}$ and $71 \%, 48 \%$, and $35 \%$ for the OS rate at 3 -yr, respectively, which were generally comparable to ours as well.

Notably, female recipients had better DFS and OS than male recipients in multivariable analyses in the present study. These results are similar to those reported by Kim et al. [17], who evaluated data from 11,797 patients transplanted from 2008 to 2010 to see how recipient-donor sex affected the outcome. They found that male recipients had worse OS and PFS compared to female recipient independent of donor sex, and that male recipients with female donors were associated with increased NRM and chronic GVHD. The latter finding is similar to that in the present study-female-to-male combination exhibited a significantly worse GRFS. 
Table 3. Multivariable analyses for survival.

\begin{tabular}{|c|c|c|c|c|c|c|c|}
\hline Parameters & $\mathrm{N}$ & $\begin{array}{c}\text { Hazard ratio for GRFS } \\
\qquad(95 \% \mathrm{Cl})\end{array}$ & $P$ & $\begin{array}{l}\text { Hazard ratio for DFS } \\
\qquad(95 \% \mathrm{Cl})\end{array}$ & $P$ & $\begin{array}{l}\text { Hazard ratio for OS } \\
\quad(95 \% \mathrm{Cl})\end{array}$ & $P$ \\
\hline \multicolumn{8}{|l|}{ Sex } \\
\hline Male & 80 & & & 1 & & 1 & \\
\hline Female & 73 & & & $0.60(0.39-0.93)$ & 0.024 & $0.56(0.35-0.90)$ & 0.017 \\
\hline \multicolumn{8}{|c|}{ Female-to-male combination } \\
\hline No & 129 & 1 & & & & & \\
\hline Yes & 24 & $1.82(1.12-2.97)$ & 0.015 & & & & \\
\hline HCT-Cl scores & 153 & & & $1.26(1.00-1.58)$ & 0.053 & $1.28(1.00-1.65)$ & 0.051 \\
\hline \multicolumn{8}{|l|}{ Disease risk index } \\
\hline Low/intermediate & 115 & & & 1 & & 1 & \\
\hline High/very high & 38 & & & $1.77(1.11-2.83)$ & 0.017 & $1.94(1.19-3.18)$ & 0.008 \\
\hline \multicolumn{8}{|l|}{ Donor type } \\
\hline MUD & 55 & 1 & & 1 & & 1 & \\
\hline HID & 62 & $1.05(0.63-1.73)$ & 0.860 & $0.89(0.54-1.47)$ & 0.645 & $0.92(0.53-1.59)$ & 0.772 \\
\hline PUD & 36 & $1.34(0.82-2.21)$ & 0.247 & $0.84(0.46-1.52)$ & 0.557 & $0.88(0.46-1.67)$ & 0.691 \\
\hline $\mathrm{CD} 4^{+}$cells in $\times 10^{6} / \mathrm{kg}$ & 153 & & & $0.91(0.83-1.00)$ & 0.042 & $0.91(0.82-1.00)$ & 0.050 \\
\hline \multicolumn{8}{|l|}{ Methotrexate use } \\
\hline Yes & 95 & 1 & & & & & \\
\hline No & 58 & $1.58(1.02-1.58)$ & 0.040 & & & & \\
\hline
\end{tabular}

Abbreviations: DFS, disease-free survival; GRFS, graft-vs.-host disease-free, relapse-free survival; HCT-Cl, hematopoietic cell transplantation comorbidity index; HID, haploidentical familial donors; MUD, matched unrelated donors; OS, overall survival; PUD, partially matchedunrelated donors.

ATG is one of the two main platforms for GVHD prophylaxis along with PTCy. It has been shown to be protective against GVHD in HCT from MUDs [18] and MSDs [19] and has been successfully incorporated into protocols for T-cell replete HCT from HIDs [8, 20, 21]. Among these, RIC with busulfan, fludarabine, and ATG for HCT from HIDs has shown promising results in terms of consistent engraftment and low rates of GVHD and NRM [22, 23].

Interestingly, the frequencies of acute GVHD of grade III-IV and chronic GVHD requiring systemic therapy were numerically higher and lower in the PUD group than in the HID and MUD groups, respectively, but the difference was not statistically significant. Raiola et al. [24] noted a more frequent occurrence of acute GVHD of grade III-IV with PUDs than with MUDs and HIDs in a heterogenous population with various hematologic malignancies. Two recent large studies, in contrast, found no difference in the incidence of acute GVHD among PUDs, MUDs, and HIDs in a population with acute leukemia [15, 25]. A future prospective trial is needed to confirm whether the risk of acute GVHD is higher with HCT from PUDs than from other donor types.

Our study has several limitations. First, the retrospective nature of the study and limited number of patients included may render this analysis hypothesis-generating at most and subtle differences likely went unnoticed. Second, the follow-up period of the study population was relatively short, and the long-term effect of the treatment remains to be evaluated. Finally, the present study did not address a different dosage of ATG, however previous reports have suggested that different doses of ATG have a differential impact on the outcome [26]. Despite these limitations, the study population was highly homogenous, in terms of conditioning regimen and use of peripheral blood as graft source, which enabled us to compare different donor types independent of these confounders.

In conclusion, the present study suggests that T-cell replete HCT from HIDs using an ATG-containing RIC regimen may be a reasonable option in the absence of MRDs in patients with acute leukemia or MDS.

\section{Authors' Disclosures of Potential Conflicts of Interest}

No potential conflicts of interest relevant to this article were reported.

\section{REFERENCES}

1. Sasazuki T, Juji T, Morishima Y, et al. Effect of matching of class I HLA alleles on clinical outcome after transplantation of hematopoietic stem cells from an unrelated donor. Japan Marrow Donor Program. N Engl J Med 1998;339:1177-85.

2. Copelan EA. Hematopoietic stem-cell transplantation. N Engl J Med 2006;354:1813-26.

3. Barker JN, Krepski TP, DeFor TE, Davies SM, Wagner JE, Weisdorf DJ. Searching for unrelated donor hematopoietic stem cells: availability and speed of umbilical cord blood versus bone marrow. Biol Blood Marrow Transplant 2002;8:257-60.

4. Ciurea SO, Bayraktar UD. "No donor"? Consider a haploidentical transplant. Blood Rev 2015;29:63-70. 
5. Powles RL, Morgenstern GR, Kay HE, et al. Mismatched family donors for bone-marrow transplantation as treatment for acute leukaemia. Lancet 1983;1:612-5.

6. Beatty PG, Clift RA, Mickelson EM, et al. Marrow transplantation from related donors other than HLA-identical siblings. N Engl J Med 1985;313:765-71.

7. Passweg JR, Baldomero H, Bader P, et al. Use of haploidentical stem cell transplantation continues to increase: the 2015 European Society for Blood and Marrow Transplant activity survey report. Bone Marrow Transplant 2017;52:811-7.

8. Luo Y, Xiao H, Lai X, et al. T-cell-replete haploidentical HSCT with low-dose anti-T-lymphocyte globulin compared with matched sibling HSCT and unrelated HSCT. Blood 2014;124:2735-43.

9. Glucksberg H, Storb R, Fefer A, et al. Clinical manifestations of graft-versus-host disease in human recipients of marrow from HL-A-matched sibling donors. Transplantation 1974;18:295-304.

10. Filipovich AH, Weisdorf D, Pavletic $S$, et al. National Institutes of Health consensus development project on criteria for clinical trials in chronic graft-versus-host disease: I. diagnosis and staging working group report. Biol Blood Marrow Transplant 2005;11: 945-56.

11. Sorror ML, Maris MB, Storb R, et al. Hematopoietic cell transplantation (HCT)-specific comorbidity index: a new tool for risk assessment before allogeneic HCT. Blood 2005;106:2912-9.

12. Armand P, Kim HT, Logan BR, et al. Validation and refinement of the Disease Risk Index for allogeneic stem cell transplantation. Blood 2014;123:3664-71.

13. Holtan SG, DeFor TE, Lazaryan A, et al. Composite end point of graft-versus-host disease-free, relapse-free survival after allogeneic hematopoietic cell transplantation. Blood 2015;125: 1333-8.

14. Solh $\mathrm{M}$, Zhang $\mathrm{X}$, Connor $\mathrm{K}$, et al. Factors predicting graftversus-host disease-free, relapse-free survival after allogeneic hematopoietic cell transplantation: multivariable analysis from a single center. Biol Blood Marrow Transplant 2016;22:1403-9.

15. Piemontese S, Ciceri F, Labopin M, et al. A comparison between allogeneic stem cell transplantation from unmanipulated haploidentical and unrelated donors in acute leukemia. J Hematol Oncol 2017;10:24.

16. McCurdy SR, Kanakry JA, Showel MM, et al. Risk-stratified outcomes of nonmyeloablative HLA-haploidentical BMT with high-dose posttransplantation cyclophosphamide. Blood 2015; 125:3024-31.

17. Kim HT, Zhang MJ, Woolfrey AE, et al. Donor and recipient sex in allogeneic stem cell transplantation: what really matters. Haematologica 2016;101:1260-6.

18. Socié G, Schmoor C, Bethge WA, et al. Chronic graft-versus-host disease: long-term results from a randomized trial on graft-versushost disease prophylaxis with or without anti-T-cell globulin ATG-Fresenius. Blood 2011;117:6375-82.

19. Kröger N, Solano C, Bonifazi F. Antilymphocyte globulin for chronic graft-versus-host disease. N Engl J Med 2016;374:1894-5.

20. Di Bartolomeo P, Santarone S, De Angelis G, et al. Haploidentical, unmanipulated, G-CSF-primed bone marrow transplantation for patients with high-risk hematologic malignancies. Blood 2013; 121:849-57.

21. Wang Y, Liu QF, Xu LP, et al. Haploidentical vs identical-sibling transplant for AML in remission: a multicenter, prospective study. Blood 2015;125:3956-62.

22. Lee $\mathrm{KH}$, Lee JH, Lee JH, et al. Reduced-intensity conditioning therapy with busulfan, fludarabine, and antithymocyte globulin for HLA-haploidentical hematopoietic cell transplantation in acute leukemia and myelodysplastic syndrome. Blood 2011;118: 2609-17.

23. Lee $\mathrm{KH}$, Lee JH, Lee JH, et al. Reduced-intensity conditioning with busulfan, fludarabine, and antithymocyte globulin for hematopoietic cell transplantation from unrelated or haploidentical family donors in patients with acute myeloid leukemia in remission. Biol Blood Marrow Transplant 2017;23:1555-66.

24. Raiola AM, Dominietto A, di Grazia C, et al. Unmanipulated haploidentical transplants compared with other alternative donors and matched sibling grafts. Biol Blood Marrow Transplant 2014;20:1573-9.

25. Versluis J, Labopin M, Ruggeri A, et al. Alternative donors for allogeneic hematopoietic stem cell transplantation in poor-risk AML in CR1. Blood Adv 2017;1:477-85.

26. Chang YJ, Wang Y, Mo XD, et al. Optimal dose of rabbit thymoglobulin in conditioning regimens for unmanipulated, haploidentical, hematopoietic stem cell transplantation: Long-term outcomes of a prospective randomized trial. Cancer 2017;123: 2881-92. 